Western University

Scholarship@Western

Oncology Publications

Oncology Department

10-21-2009

\title{
The effects of field-of-view and patient size on CT numbers from cone-beam computed tomography.
}

Katrina Y T Seet

Arvand Barghi

Slav Yartsev

Jacob Van Dyk

The University of Western Ontario, vandyk@uwo.ca

Follow this and additional works at: https: / ir.lib.uwo.ca/oncpub

Part of the Medical Biophysics Commons, and the Oncology Commons

Citation of this paper:

Seet, Katrina Y T; Barghi, Arvand; Yartsev, Slav; and Van Dyk, Jacob, "The effects of field-of-view and patient size on CT numbers from cone-beam computed tomography." (2009). Oncology Publications. 123.

https://ir.lib.uwo.ca/oncpub/123 
The effects of field-of-view and patient size on CT numbers from cone-beam computed tomography

This content has been downloaded from IOPscience. Please scroll down to see the full text. 2009 Phys. Med. Biol. 546251

(http://iopscience.iop.org/0031-9155/54/20/014)

View the table of contents for this issue, or go to the journal homepage for more

Download details:

IP Address: 129.100.104.78

This content was downloaded on 09/02/2017 at 15:23

Please note that terms and conditions apply.

You may also be interested in:

CBCT: dosimetric effects of $\mathrm{HU}$ number to electron density calibration Joan Hatton, Boyd McCurdy and Peter B Greer

CBCT scatter kernel estimation with an ESF method

Heng Li, Radhe Mohan and X Ronald Zhu

Evaluation of on-board $\mathrm{kV}$ cone beam CT (CBCT)-based dose calculation

Yong Yang, Eduard Schreibmann, Tianfang Li et al.

Prior image constrained scatter correction

Stephen Brunner, Brian E Nett, Ranjini Tolakanahalli et al.

Dose calculation accuracy using CBCT for pelvic adaptive radiotherapy Huaiqun Guan and Hang Dong

Beam characteristics and radiation output of a kilovoltage cone-beam CT George X Ding and Charles W Coffey

Characteristics of kilovoltage $x$-ray beams used for cone-beam CT in radiation therapy

George X Ding, Dennis M Duggan and Charles W Coffey

Reduction in $\mathrm{x}$-ray scatter and radiation dose for VOI cone-beam breast CT

Chao-Jen Lai, Lingyun Chen, Huojun Zhang et al.

Reconstructing cone-beam CT with spatially varying qualities for adaptive radiotherapy: a proof-of-principle study Wenting Lu, Hao Yan, Xuejun Gu et al. 


\title{
The effects of field-of-view and patient size on CT numbers from cone-beam computed tomography
}

\author{
Katrina Y T Seet, Arvand Barghi, Slav Yartsev and Jake Van Dyk \\ London Regional Cancer Program, London Health Sciences Centre, London, Ontario, Canada \\ E-mail: slav.yartsev@lhsc.on.ca
}

Received 21 January 2009, in final form 23 August 2009

Published 1 October 2009

Online at stacks.iop.org/PMB/54/6251

\begin{abstract}
Cone-beam computed tomography (CBCT) is used for patient alignment before treatment and is ideal for use in adaptive radiotherapy to account for tumor shrinkage, organ deformation and weight loss. However, CBCT images are prone to artifacts such as streaking and cupping effects, reducing image quality and CT number accuracy. Our goal was to determine the optimum combination of cone-beam imaging options to increase the accuracy of image CT numbers. Several phantoms with and without inserts of known relative electron densities were imaged using the Varian on-board imaging system. It was found that CT numbers are most influenced by the selection of field-of-view and are dependent on object size and filter type. Image acquisition in half-fan mode consistently produced more accurate CT numbers, regardless of phantom size. Values measured using full-fan mode can differ by up to $7 \%$ from planning $\mathrm{CT}$ values. No differences were found between CT numbers of all phantom images with low and standard dose modes.
\end{abstract}

\section{Introduction}

Conventionally, patients are aligned to external markings made at the time of radiotherapy simulation. It may be sufficient if the target and organs-at-risk have not moved relative to external markings. Now, imaging devices such as electronic portal imaging and flatpanel detectors are available where orthogonal, two-dimensional images can be acquired to evaluate setup correctness. More recently, cone-beam computed tomography (CBCT) has been developed as a three-dimensional imaging method for image-guided radiotherapy where patients are imaged and repositioned in reference to the target volume (Jaffray and Siewerdsen 2000, Li et al 2006, Fu et al 2007, Ding et al 2006, Hansen et al 2006).

Cone-beam imaging is currently available on the Elekta Synergy and Varian On-board imaging (OBI) systems. These systems have a $\mathrm{kV}$ x-ray source and a flat-panel detector mounted orthogonal to the megavoltage (MV) beam of the linear accelerator. Such an 
arrangement allows convenient patient imaging before treatment and minimizes patient motion between patient alignment and treatment. A cone-shaped $\mathrm{kV}$ beam rotates around the patient (usually $360^{\circ}$ rotation but the range can be varied at setup) and 360 or 720 projections are recorded and reconstructed. Although CBCT imaging can greatly improve patient treatment accuracy by positioning, significant changes (5-8\%) in the delivered treatment have been found for sessions where there were large anatomic variations caused by setup inaccuracy, organ deformation, tumor shrinkage and/or weight loss (Lee et al 2008). In the case when such changes may be clinically significant, original radiotherapy plan should be adapted or modified to conform to the current (e.g. CBCT) images prior to further treatment to ensure that the intended dose delivery is achieved (Karellas et al 2008). Several studies on dose calculations based on CBCT images have been conducted investigating the feasibility of cone-beam-based plans for dosimetry calculations (Lee et al 2007, 2008, Ding et al 2007b, Nijkamp et al 2008, Yang et al 2007, Yoo and Yin 2006, Richter et al 2008). Currently, CBCT images are prone to artifacts such as streaking and cupping effects, reducing image quality and CT number accuracy (Ding et al 2007a, Katsumata et al 2006). This is due to increased detected scatter, which is a direct consequence of the large field cone-beam design. For reliable dosimetry calculations, an accurate conversion of CT numbers (HU) to electron density (ED) is required. Due to scatter dependence on factors such as patient size, complexity of patient composition and incoming x-ray characteristics, it may not be viable to use a single standard HU-ED calibration curve. For example, Cozzi et al showed that a difference of approximately $300 \mathrm{HU}$ in CT number lead to a maximum treatment dose error of $2.5 \%$ (Cozzi et al 1998). Previous studies have shown the mean dose can vary by up to $3.6 \%$ between CBCT and planning CT (pCT)-based treatment plans (Lee et al 2008, Yoo and Yin 2006). Plans based on helical fan-beam CTs are still far superior to CBCT-based dosimetry (Lo et al 2005 ) with mean dose differences in calculations for a head and neck case of only $0.3 \pm 0.3 \%$ (Liang et al 2008).

In CBCT, detected photon scatter is significantly greater than in the fan-beam geometry. This results in lower image quality. Previously, Guan et al (2002) investigated the effect of altering $\mathrm{kV}, \mathrm{mA}$ and slice thickness in $\mathrm{kVCT}$ on the CT numbers. Energy settings were found to have the greatest impact on CT numbers, although not clinically significant, i.e. less than $2 \%$ variation between HU-ED calibration curves. However, thus far, the effects of field-of-view (FOV) and filter type in CBCT imaging have not been reported. This paper considers for the first time the variations in CT numbers due to CBCT filter, FOV and dose settings on the Varian OBI system.

\section{Materials and methods}

\subsection{Computed tomography}

The Varian OBI system includes a $\mathrm{kV}$ x-ray source and a $\mathrm{kV}$ x-ray detector mounted on the linear accelerator orthogonal to the MV treatment-beam axis. Images were obtained using the low $(125 \mathrm{kVp}, 40 \mathrm{~mA}$ and $10 \mathrm{~ms})$ and standard $(125 \mathrm{kVp}, 80 \mathrm{~mA}$ and $25 \mathrm{~ms})$ dose settings and reconstructed with the $512 \times 512$ matrix in axial slices. An anti-scatter grid is permanently installed on the flat-panel detector. An aluminum bow-tie filter is used for all image acquisitions. This filter reduces scattering effects e.g. reduces beam hardening and charge trapping in the detector (Mail et al 2009, Siewerdsen and Jaffray 2001). It also compensates for the heel effect where flux is equalized at the detector (Ding et al 2007a, Glover 1982). Overall, the filter reduces the skin dose to the patient and improves image quality (Siewerdsen and Jaffray 2001). The filters are $1.5 \mathrm{~cm}$ thick at the center. The 


\section{Full fan}

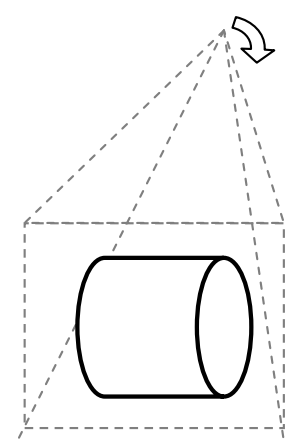

Lateral view
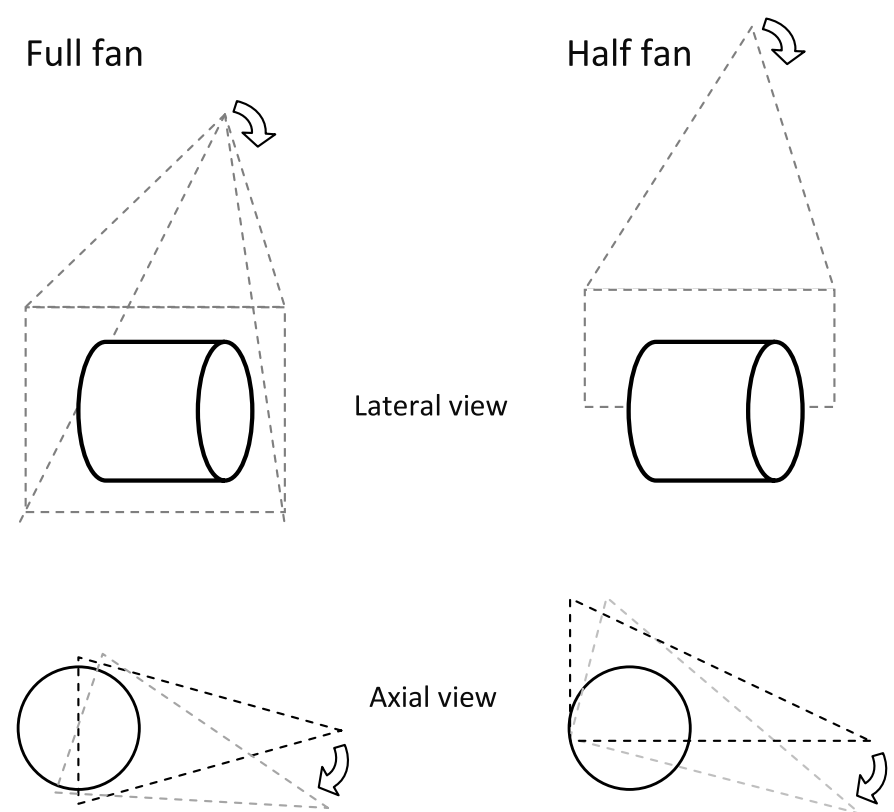

Figure 1. Beam positioning of full and half-fan scans. A symmetric square-cone of kV x-rays rotates about the isocenter for cone-beam imaging in full-fan mode. In half-fan mode, the position of the $\mathrm{kV}$ source remains the same, however, half the beam is truncated by an aluminum filter.

half-bow-tie is used when either the left-right (LR) or posterior-anterior (PA) dimension exceeds $25 \mathrm{~cm}$, since the reconstruction uses the largest dimension for its FOV. Conversely, a full-bow-tie filter is used for diameters less than or equal to $25 \mathrm{~cm}$. The FOV in the craniocaudal direction is fixed at 15 and $14 \mathrm{~cm}$ for full- and half-fan modes shown in figure 1 . For each of these scanning modes, the jaw settings were $X 1=X 2=13.6 \mathrm{~cm}$ and $Y 1=Y 2=10.7$ $\mathrm{cm}$ for full-fan mode and $X 1=6.8 \mathrm{~cm}, X 2=23.5 \mathrm{~cm}, Y 1=Y 2=10.3 \mathrm{~cm}$ for half-fan mode scans. Planning CT (pCT) studies were acquired on a CT simulator (Philips, Brilliance CT Big Bore) at $120 \mathrm{kV}, 85 \mathrm{~mA}$ and $3 \mathrm{~mm}$ slice spacing.

\subsection{Cylindrical phantoms}

Four homogeneous cylindrical phantoms of known densities were used to test the consistency of CT numbers obtained by the OBI system. The first is a $20 \mathrm{~cm}$ diameter phantom, commonly used for the calibration of cone-beam systems (Catphan 504, The Phantom Laboratory, New York, NY). The phantom has several sections, which include a uniform, water-equivalent section, and an inhomogeneous section. The second and the third phantoms are $30 \mathrm{~cm}$ solidwater phantoms (Gammex-RMI, Middletown, WI). These phantoms are commonly used for the calibration of the tomotherapy system. Each is identical except one that includes inserts of various electron densities for HU-ED calibration within a homogenous water-equivalent material. In-house cylindrical perspex phantoms (density $=1.19 \mathrm{~g} \mathrm{~cm}^{-3}$, expected CT number $=100 \mathrm{HU}$ ) were also imaged. $\mathrm{pCT}$ and CBCT studies of all phantoms were exported to the Pinnacle ${ }^{3}$ (version $8.0 \mathrm{~m}$ ) treatment planning system (Philips Healthcare, Fitchburg, WI, USA) as DICOM files. 

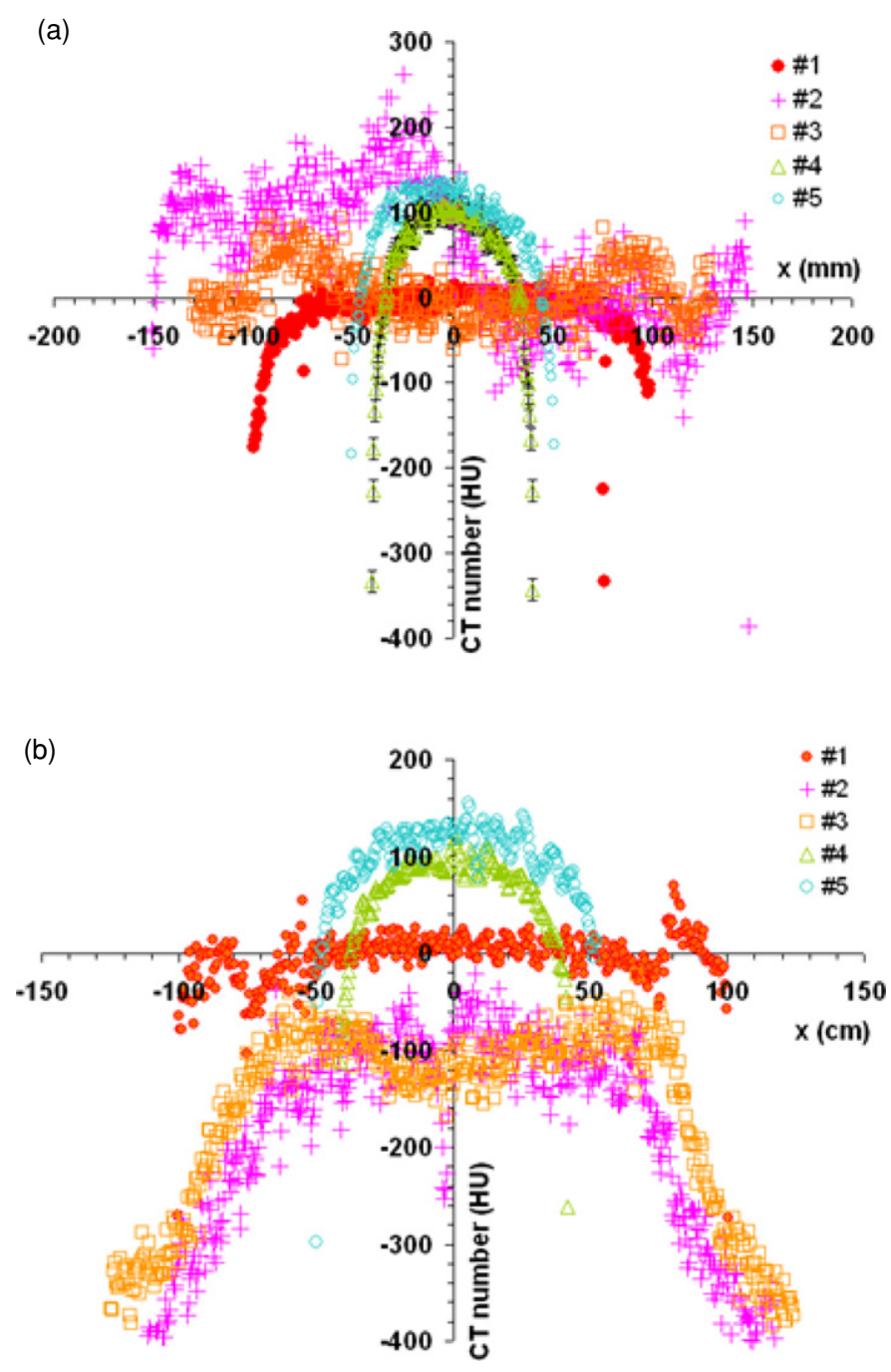

Figure 2. CT numbers along the lateral $(x)$ axis of uniform-density phantoms of various sizes. Images were acquired with (a) half-fan and (b) full-fan bow-tie filters. Phantoms $1(D=20 \mathrm{~cm})$, $2(D=30 \mathrm{~cm})$ and $3(D=30 \mathrm{~cm})$ are composed of a water-equivalent material. Phantoms 4 and 5 are perspex phantoms $(D=8$ and $10 \mathrm{~cm})$.

\section{Results}

\subsection{Uniform-density phantoms}

All uniform density phantoms were imaged in full- and half-fan modes. The OBI system limits the use of each filter to FOV $<25 \mathrm{~cm}$ for full-fan mode and FOV $\geqslant 26 \mathrm{~cm}$ for halffan mode. Therefore, to restrict the effects of any HU changes to filter-type only, 25 and $26 \mathrm{~cm}$ FOV settings were used for full- and half-fan scans, respectively. CT numbers across phantoms were measured using Image J $1.40 \mathrm{~g}$ (NIH, Bethesda, MD, USA). In the analyzed image, each data point represents the CT number per voxel. The voxel size is $(\mathrm{FOV} / 512)^{2} \times$ slice thickness. The CT number profiles of the two phantoms imaged with half- and full-fan modes are presented in figures 2(a) and (b), respectively. Five phantoms are listed. Phantom 
Table 1. Average of CT numbers across uniform water-equivalent cylindrical phantoms obtained using full- and half-fan filters (see section 3.1 for acquisition details).

\begin{tabular}{llllccc}
\hline & & & & \multicolumn{2}{c}{ Measured HU (\% difference) } \\
\cline { 4 - 6 } No & $D(\mathrm{~cm})$ & $L(\mathrm{~cm})$ & $\mathrm{HU}_{\text {exp }}($ bulk) & $\mathrm{pCT}$ & Full-fan & Half-fan \\
\hline 1 & 20 & 20 & $0-20$ & $19 \pm 7$ & $8.8 \pm 7.9(-0.5 \pm 0.3)$ & $0.54 \pm 5.7(-1.0 \pm-9.6)$ \\
2 & 30 & 18 & $0-20$ & $20 \pm 18$ & $-98 \pm 43(-5.9 \pm 5.6)$ & $38 \pm 58(0.9 \pm 5.2)$ \\
3 & 30 & 18 & $0-20$ & $20 \pm 18$ & $-123 \pm 17(-7.2 \pm 2.7)$ & $-18 \pm 22(-1.9 \pm 4.3)$ \\
4 & 8 & 12 & 100 & $127 \pm 3$ & $94 \pm 14(-0.3 \pm 0.1)$ & $102 \pm 5(-0.2 \pm 0.1)$ \\
5 & 10 & 15 & 100 & $124 \pm 3$ & $123 \pm 9(0.0 \pm 0.1)$ & $125 \pm 6(0.0 \pm 0.1)$ \\
\hline
\end{tabular}

1 is the $20 \mathrm{~cm}$ diameter phantom, phantom 2 is the $30 \mathrm{~cm}$ diameter phantom, phantom 3 is phantom 2 rotated $90^{\circ}$, phantoms 4 and 5 are 8 and $10 \mathrm{~cm}$ diameter cylinders of perspex. The truncation of some data points can be observed in cases where phantom diameter is greater than the FOV e.g. phantom 2 imaged in full-fan mode. Average CT numbers measured across the phantoms are shown in table 1 and a percentage difference from pCT-image measurements are shown in parentheses. All presented results were acquired using the standard dose mode as no difference was found between images obtained using low or standard dose modes for all five acquisitions. No significant changes were observed in phantom 1 between filter types. However, significant differences were measured for the larger $30 \mathrm{~cm}$ phantom (phantom 2). Full-fan mode underestimates CT numbers through the center of the phantom, while halffan mode causes a large fluctuation of CT numbers. In half-fan mode, CT numbers across phantom 2 vary by $150 \mathrm{HU}$ between two sides of the phantom. These fluctuations do not occur when the phantom is rotated (phantom 3). Previous literature has also observed profiles fluctuations of homogeneous water phantoms of $D=16.0$ and $26.6 \mathrm{~cm}$ (Yang et al 2007).

The CT numbers across the perspex phantoms ( 4 and 5) were consistent and accurate both in full- and half-fan modes. For perspex, CT numbers are expected to be $100 \mathrm{HU}$. Phantom 4 showed a mean value of $94 \pm 14$ and $102 \pm 5 \mathrm{HU}$ when scanned in full- and half-fan modes, respectively. Phantom 5 showed average values of $123 \pm 9$ and $125 \pm 6 \mathrm{HU}$ when scanned in full- and half-fan modes, respectively, which is in good agreement (within 1\%) with pCT values.

Switching the filter from half-fan to full-fan mode decreases the area of reconstruction in the axial plane by $50 \mathrm{~cm}^{2}$. To investigate the exclusion of scattering materials from the reconstruction area due to an adequate FOV, images were acquired using FOV 15-26 cm. The profiles across the $20 \mathrm{~cm}$ diameter phantom are illustrated in figure 3 . A decrease in the average CT number was measured for FOV $\leqslant 20 \mathrm{~cm}$. Measurements were also repeated for the $30 \mathrm{~cm}$ phantom where a FOV $\leqslant 25 \mathrm{~cm}$ resulted in inaccurate CT numbers.

Two peaks at $\pm 80 \mathrm{~mm}$ are the result of a crescent artifact caused by a shift of the bowtie profile from one projection to the next. The manufacturer claims this is due to minor mechanical instabilities e.g. tilted source or focal spot shift (Varian Medical Systems, Inc. 2008). The prominence of this feature is more noticeable in full-fan images; however, it also occurs in half-fan mode where CT numbers suddenly change.

\subsection{Phantoms with inserts of different densities}

The effect of FOV on the measurement of CT numbers on two phantoms with different diameters was investigated. CT numbers of inserts with known electron densities were imaged using CBCT. Image J $1.40 \mathrm{~g}$ was used to measure average CT numbers within a 


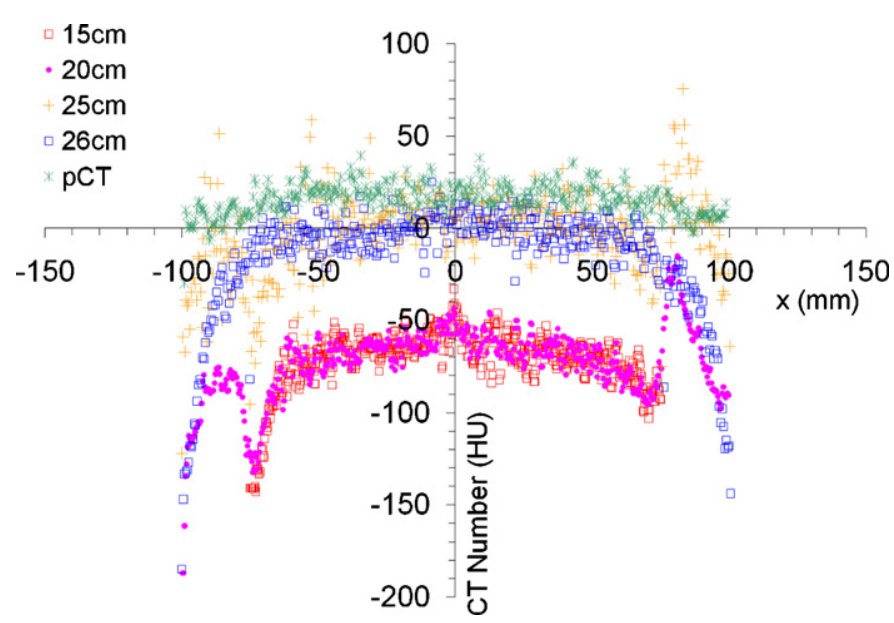

Figure 3. Profile of CT numbers from CBCT images along the lateral $(x)$ axis of phantom $1(D=$ $20 \mathrm{~cm}$ ) taken with various fields-of-view (full-fan mode for FOV $\leqslant 25 \mathrm{~cm}$ diameter and half-fan mode for FOV $\geqslant 26 \mathrm{~cm})$. CT numbers determined from $\mathrm{pCT}$ images are also shown $(23 \mathrm{~cm} \mathrm{FOV})$.

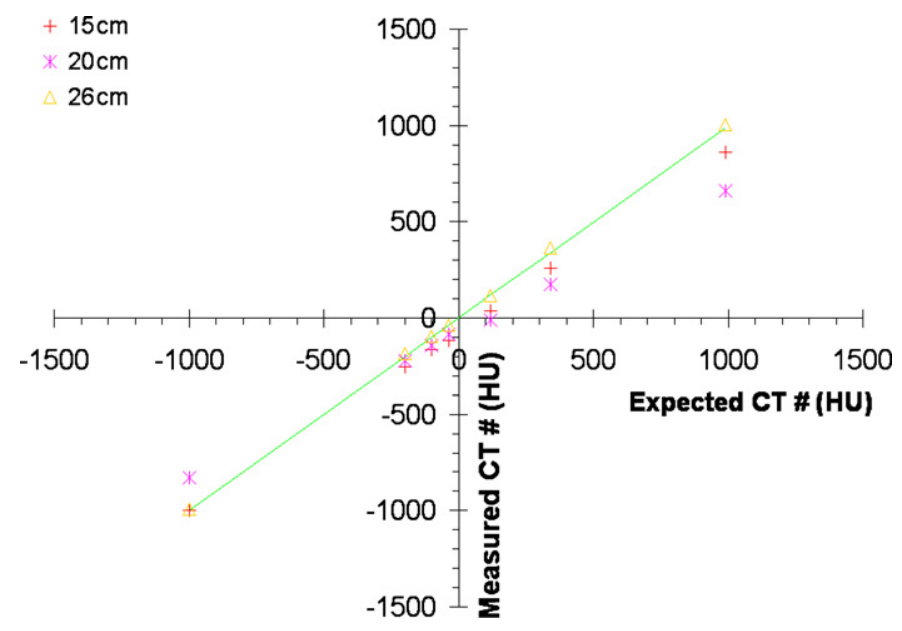

Figure 4. CT numbers of inserts in the $20 \mathrm{~cm}$ phantom imaged using 15, 20 and $26 \mathrm{~cm}$ FOVs.

circular region-of-interest (ROI) of an axial image slice. The region selected was within an area of uniform electron density, corresponding to the cross-section of a RED insert. Regions of diameters 0.8 and $2.0 \mathrm{~cm}$ were used for RED inserts in the 20 and $30 \mathrm{~cm}$ diameter phantoms, respectively. The measured CT values of inserts within the smaller phantom are illustrated in figure 4. The solid line represents expected values. Errors due to noise are not shown, as they are smaller than the marker size. Scans obtained using a FOV of $26 \mathrm{~cm}$ produced the most accurate results which agree within 10\% with the manufacturer's specifications. This is in agreement with previously published measurements (Lee et al 2008, Yang et al 2007). However, the accuracy of CT numbers diminished as FOV decreased e.g. CT numbers from images obtained with a $20 \mathrm{~cm}$ FOV were less accurate than those obtained with $15 \mathrm{~cm}$ FOV. This is due to the cupping artifact, as detailed in section 4 . 


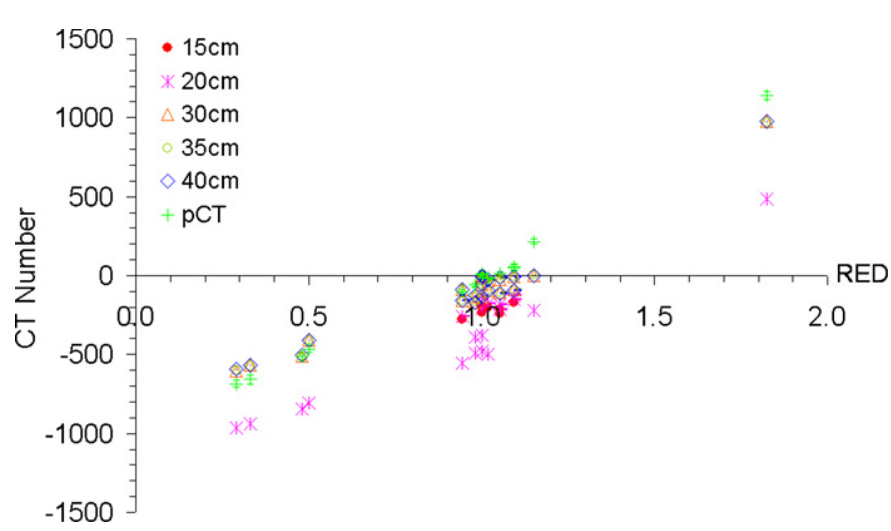

Figure 5. CT numbers of electron density inserts in the $30 \mathrm{~cm}$ phantom, measured from CBCT images acquired at various field-of-views.

The larger phantom was also imaged. Manufacturers of the $30 \mathrm{~cm}$ phantom did not provide HU values; therefore, CT numbers were determined by pCT and regarded as expected values. Five FOVs ranging from 15 to $40 \mathrm{~cm}$ were used for imaging, and the measured CT values for RED inserts are shown in figure 5. Only the errors of pCT number $(18 \pm 4 \mathrm{HU})$ are shown to avoid graph over-crowding. The average standard deviation of the CT numbers for each insert by CBCT imaging is $36 \pm 5 \mathrm{HU}$. Scans acquired with 15 and $20 \mathrm{~cm}$ FOV show the most significant difference where respective CT numbers were, on average, $240 \pm 30$ and $330 \pm 150 \mathrm{HU}$ lower than the measured pCT values. For FOVs greater than $20 \mathrm{~cm}$, average CT numbers increase by $100 \pm 60 \mathrm{HU}$ from pCT values. The phantom was also rotated by $90^{\circ}$ and imaged. In this orientation, CT numbers were on average $8 \pm 25 \%$ lower than pCT values when imaged using a FOV less than $20 \mathrm{~cm}$. This difference was reduced to $2 \pm 5 \%$ when scanned with a FOV greater than $30 \mathrm{~cm}$.

Inserts of varying electron densities were rearranged in the $30 \mathrm{~cm}$ phantom. Figure 6 illustrates these arrangements: (a) and (b) were imaged with CBCT using halffan and pCT, respectively. Average CT numbers within the inserts from CBCT and pCT scans are reported in figure 7 . Inserts located in the inner and outer regions are differentiated by marker shape. For the same insert in a different location, the CT numbers obtained from a full-fan scan varied considerably from measured pCT values, with a difference of up to $370 \pm 190 \mathrm{HU}$. A smaller difference $(130 \pm 80 \mathrm{HU})$ was observed between images scanned using half-fan mode. The greatest difference $(1080 \pm 70 \mathrm{HU})$ was found between CBCT and pCT values for the high-density cortical bone $\left(1.824 \mathrm{~g} \mathrm{~cm}^{-3}\right)$ obtained by full-fan mode for the phantom in orientation shown in figure 6(b). With the exception of cortical bone, inserts located in the inner region (within an $8 \mathrm{~cm}$ radius from the isocenter) were measured to be 200-300 HU lower than expected CT numbers. For inserts in the outer region of the phantom, the inaccuracy of CT numbers doubled. The causes of these affects are discussed below.

The orientation of the phantom also affected CT numbers of inserts. The average percentage difference between CT numbers of inserts obtained by CBCT of phantom 2 (see table 1) with respect to pCT values was $940 \%$, while it was $840 \%$ for phantom 3 . These values decreased to $260 \%$ and $30 \%$, respectively, when scanned in the half-fan mode. These results suggest that the combination of the location of the high-density cortical bone at the outer edge of the phantom and scanning in half-fan mode produces less scattering artifacts. 

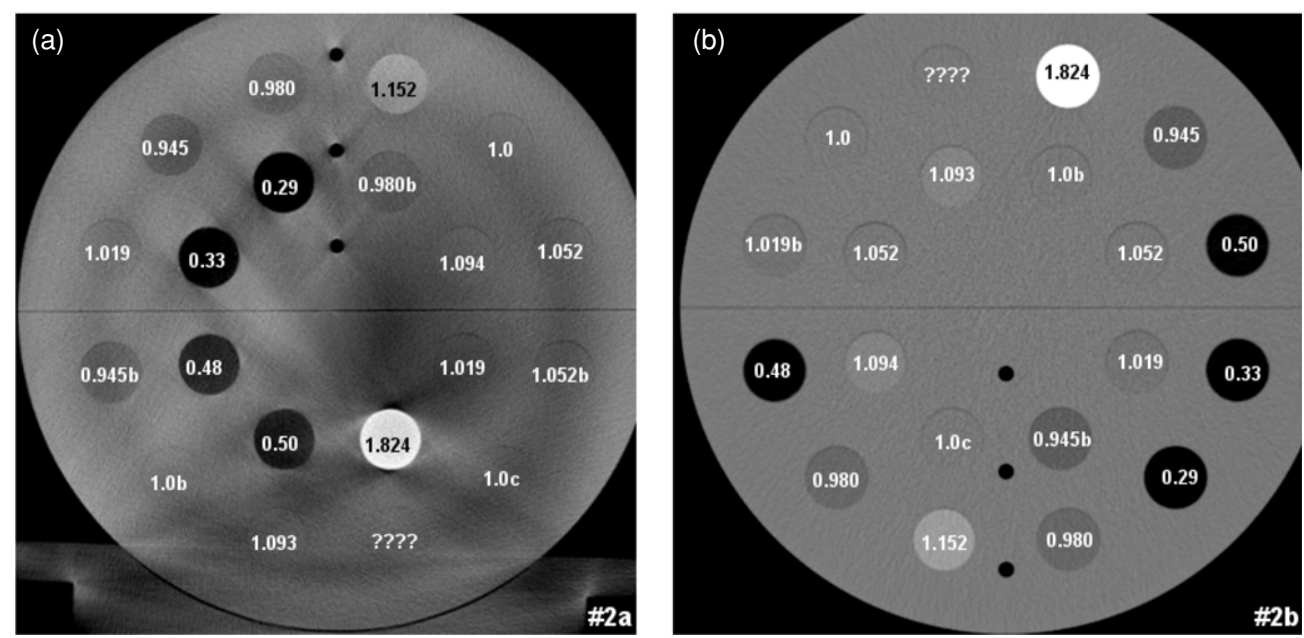

Figure 6. Location of inserts of known relative electron densities (REDs) in the $30 \times 18 \mathrm{~cm}^{2}$ water-equivalent phantom: (a) phantom 2a imaged with CBCT and (b) phantom $2 \mathrm{~b}$ imaged by pCT. Images were reconstructed using an external image processing program (Image $\mathrm{J} 1.40 \mathrm{~g}$, National Institutes of Health, MD, USA).

\section{Discussion}

Although there are other systems which produce far better image quality than CBCT, the distinct advantage of on-board CBCT imaging is the 'in-room' acquisition ability where the patients are imaged in their treatment position. This provides minimal patient movement, low dose, rapid acquisition and reconstruction. CBCT enables frequent imaging, hence is a precursor for adaptive planning. This paper reports on the optimization of imaging settings available to radiation therapists to obtain the most accurate results for adaptive planning.

Pronounced streaking artifacts are observed in figure 6(a) as an illustration of how the complexity and size of patients can significantly affect CT numbers obtained by CBCT. This is more pronounced in the case of high-density materials. For the high-density cortical bone (see figure 1(a)), the large difference between CBCT and pCT numbers is a consequence of streaking artifacts producing partial volume effects for voxels within homogenous inserts. CT numbers are most accurate if using a FOV greater than the patient diameter. All previous publications reported investigations using a $D=L=20 \mathrm{~cm}$ cylindrical phantom (phantom 1) with inserts of known electron densities (Yoo and Yin 2006, Ding et al 2007a, Lee et al 2008). Full- and half-fan scans were reported to produce CT numbers within 5\% of accepted values. These scans were performed with a FOV of $45 \mathrm{~cm}$ for half-fan and $25 \mathrm{~cm}$ for full-fan mode. For adaptive planning, it may not be optimal to use the maximum FOV as the spatial resolution is compromised. Another concern is the positioning of collimating blades. In the Varian OBI system, these blades have a fixed field size for both full- and half-fan modes independent of the FOV used for image reconstruction. Therefore, the FOV change in this system does not affect patient imaging dose.

The manufacturer recommends using a FOV equal to the maximum patient dimension. This prevents the truncation of data in axial slice, where cupping is known to produce artifacts with a drop in CT number values. Effects are more prominent in CBCT imaging than fanbased imaging due to the increased detected scatter (Siewerdsen and Jaffray 2001, Yoo and 


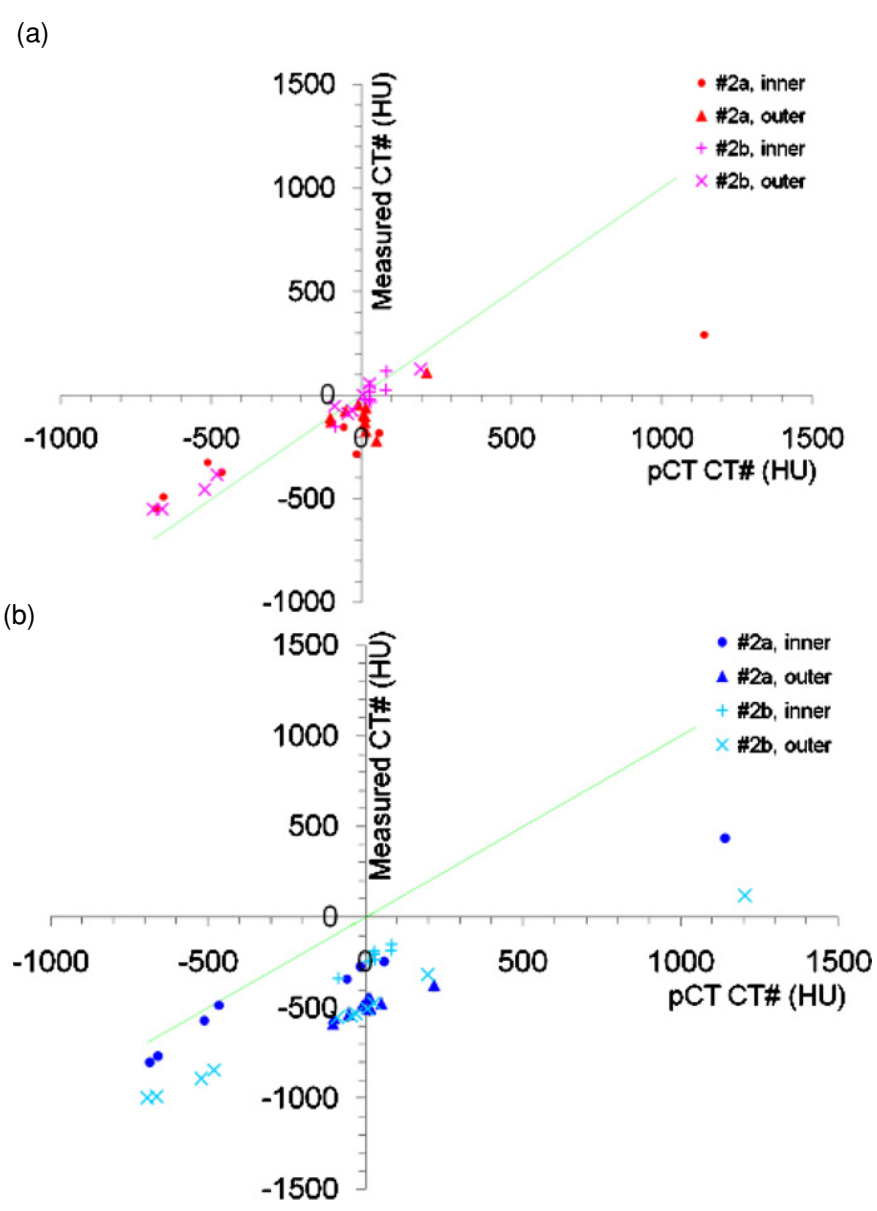

Figure 7. CT numbers of inserts measured in rotated phantoms, acquired by CBCT using (a) half- and (b) full-fan modes. Inserts were rearranged at different positions (location of inserts are illustrated in figure 1). Inserts in the inner region of the phantom are within $15 \mathrm{~cm}$ diameter inner circle and outside this circle for the 'outer'. The solid line (-) represents values from planning CT (pCT).

Yin 2006, Yang et al 2007, Petit et al 2008). Previous reports on artifacts have explained these effects as due to the reconstruction algorithm's inability to properly account for objects outside the FOV (Katsumata et al 2007). Our results from imaging in the full-fan mode and $25 \mathrm{~cm}$ FOV for phantom 2 show that the average CT number is $-65 \pm 7 \mathrm{HU}$ lower than expected (see crosses in figure 3(b). The cupping artifact is not observed when the FOV is greater than $26 \mathrm{~cm}$. For a smaller phantom (phantom 1), imaging at FOV $=\mathrm{D}$ also produced cupping artifacts. Upon closer inspection of axial images, the $20 \mathrm{~cm}$ FOV truncates the phantom by the slightest fraction. A difference of $20 \mathrm{~mm}^{2}$ in the axial area was enough to produce such artifacts (see figure 8). This demonstrates the sensitivity of the cupping artifacts in CBCT when imaging in a full-fan mode. It is therefore vital, especially in adaptive planning, to use a FOV that includes the whole patient in the traverse plane.

The profiles of phantom 2 show that CT numbers fluctuate when imaged with FOVs between 26 and $40 \mathrm{~cm}$ (see figure 2(a). Yang et al (2007) noticed such fluctuations for phantoms imaged using half-fan mode and FOV $>$ D. The same fluctuation was observed in 

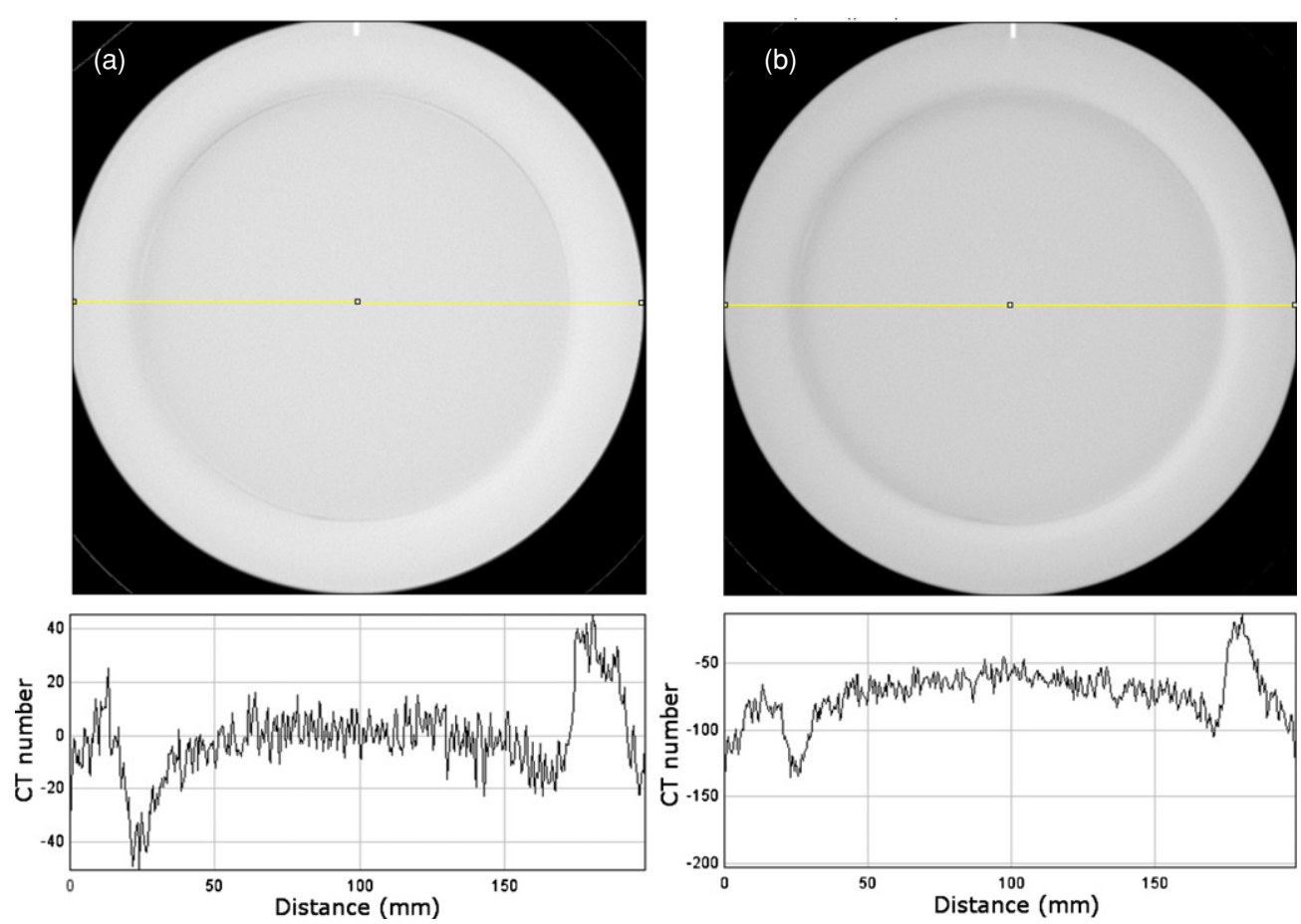

Figure 8. Axial slices from CBCT of phantom 1 where the area truncated by the FOV is (a) 5.41 and (b) $6.01 \mathrm{~cm}^{2}$, respectively. This lead to a difference of $70 \mathrm{HU}$ of the average CT number across the physical limits of the phantom.

phantom 1, although to a much smaller degree. These CT number fluctuations are caused by scattering alone and independent of data truncation in the axial plane. Such fluctuations are due to an increased influence of scatter radiation and increase with phantom size (Katsumata et al 2007). Note, however, that this effect is not directly related to diameter, as our scans of phantom $2(D=L=20 \mathrm{~cm})$ did not demonstrate such fluctuations. Nevertheless, average CT numbers across the phantom scanned using half-fan mode are more accurate than those obtained with full-fan mode.

Artifacts may also be the result of initial system calibrations where there was no option to change the FOV. The Varian OBI system performs full- and half-fan mode calibrations with 25 and $26 \mathrm{~cm}$ FOV, respectively. This places a restriction on the size of the phantom to be used for calibration.

In phantom $2 \mathrm{~b}$, the location of the cortical bone insert on the outer edge of the phantom produced less artifacts compared to positioning of this insert in the inner region. The former insert arrangement is representative of human anatomy where bones are located close to the outside edge of the body, as in the head and thorax. This is an indication that CBCT for adaptive planning may not be suitable for patients with high-density structures located within the central portion of the body.

\section{Conclusion}

In this study, images of uniform phantoms were obtained with $\mathrm{kV} \mathrm{CBCT}$ using the various settings available. Imaging phantoms with FOV less than the phantom size is known to produce cupping artifacts. Even imaging with full-fan mode and FOV equal to the phantom 
size produced CT numbers lower than expected as a result of the slight data truncation. It is vital to ensure that the FOV encloses the entire phantom or patient. The major factors which influence the precision of CT numbers are the FOV and size of the phantom. The FOV should be small to maximize spatial resolution and large enough to avoid the truncation of scattering material, particularly when imaging with a full-fan mode. Imaging with a FOV $<\mathrm{D}$ with half-fan mode appears independent of 'cupping' artifacts but demonstrates CT fluctuations of varying degrees, depending on phantom size. We recommend initial calibration of the system with a phantom of size comparable to the object to be imaged. At the current state of OBI development, CBCT is not ideal for adaptive planning due to its vulnerability to increased scatter and therefore inaccurate representation of material density, compared to planning CT. CBCT provides low-dose, regular, up-to-date images of the patient immediately prior to treatment thereby improving genuine accuracy. Adaptive planning using CBCT imaging is useful for cases where the patient anatomy has significantly changed, recognizing, however, the limitation in the accuracy of the CT number.

\section{Acknowledgment}

This study was conducted with the support of the Ontario Institute for Cancer Research through funding provided by the government of Ontario.

\section{References}

Cozzi L, Fogliata A, Buffa F and Bieri S 1998 Dosimetric impact of computed tomography calibration on a commercial treatment planning system for external radiation therapy Radiother. Oncol. 48 335-8

Ding G, Duggan D and Coffey C 2007a Characteristics of kilovoltage x-ray beams used for cone-beam computed tomography in radiation therapy Phys. Med. Biol. 52 1595-615

Ding G, Duggan D, Coffey C, Deeley M, Hallahan D, Cmelak A and Malcolm A 2007b A study on adaptive IMRT treatment planning using kV cone-beam CT Radiother. Oncol. 85 116-25

Ding G, Duggan D, Coffey C, Hallahan D and Deeley M 2006 Adaptive IMRT using cone-beam CT: a case study on patients with bulky head and neck tumors (abstract) Med. Phys. 332024

Fu W, Yang Y, Yue N J, Heron D E and Huq S M 2007 Study of rotational setup errors and their dosimetric impacts on head and neck IMRT treatments using kilovoltage cone-beam computed tomography (kV CBCT) (abstract) Int. J. Radiat. Oncol. Biol. Phys. $69 \mathrm{~S} 188$

Glover G H 1982 Compton scatter effects in CT constructions Med. Phys. 9 860-7

Guan H, Yin F and Kim J 2002 Accuracy of inhomogeneity correction in photon radiotherapy from CT scans with different settings Phys. Med. Biol. 47 N223-31

Hansen E K, Bucci M K, Quivey J M, Weinberg V and Xia P 2006 Repeat CT imaging and replanning during the course of IMRT for head-and-neck cancer Int. J. Radiat. Oncol. Biol. Phys. 64 355-62

Jaffray D and Siewerdsen J 2000 Cone-beam computed tomography with a flat-panel imager: initial performance characterization Med. Phys. 27 1311-23

Karellas A, Lo J Y and Orton C G 2008 Cone beam X-ray CT will be superior to digital X-ray tomosynthesis in imaging the breast and delineating cancer Med. Phys. 35 409-11

Katsumata A, Hirukawa A, Noujeim M, Fujishita S O M N M, Ariji E and Langlais R P 2006 Image artifact in dental cone-beam CT Oral Surg. Oral Med. Oral Pathol. 101 652-7

Katsumata A, Hirukawa A, Okumura S, Naitoh M, Fujishita M and Ariji E 2007 Effects of image artifacts on grayvalue density in limited-volume cone-beam computerized tomography Oral Surg. Oral Med. Oral Pathol. 104 $829-36$

Lee L, Le Q, La T and Xing L 2007 IMRT dose reconstruction on cone-beam computed tomography (CBCT): a platform for head-and-neck adaptive therapy (abstract) Int. J. Radiat. Oncol. Biol. Phys. 69 S412

Lee L, Le Q and Xing L 2008 Retrospective IMRT dose reconstruction based cone-beam CT and MLC log-file Int. J. Radiat. Oncol. Biol. Phys. 70 634-44

Li H, Zhu X R, Zhang L, Dong L, Ahamad A, Chao K S C, Morrison W H, Rosenthal D I, Schwartz D L and Garden A S 2006 Assessment of positioning accuracy of head and neck patients receiving IMRT using on-board imaging and cone beam CT (abstract) Int. J. Radiat. Oncol. Biol. Phys. 66 S144-5 
Liang J, Chi Y and Yan D 2008 Cone beam CT image based adaptive inverse planning for head and neck cancer: a validation study (abstract) Int. J. Radiat. Oncol. Biol. Phys. 72 S600-1

Lo T, Yang Y, Schreibniann E, Li T and Xing L 2005 Mapping electron density distribution from planning CT to cone-beam CT (CBCT): a novel strategy for accurate dose calculation based on CBCT (abstract) Int. J. Radiat. Oncol. Biol. Phys. 63 S507

Mail N, Moseley D J, Siewerdsen J H and Jaffray D A 2009 The influence of bowtie filtration on cone-beam CT image quality Med. Phys. 36 22-32

Nijkamp J, Pos F, Nuver T, de Jong R, Remeijer P, Sonke J and Lebesque J 2008 Adaptive radiotherapy for prostate cancer using kilovoltage cone-beam computed tomography: first clinical results Int. J. Radiat. Oncol. Biol. Phys. 70 75-82

Petit S F, van Elmpt W J C, Lambin P and Dekker A L A J 2008 Calibration of megavoltage conebeam CT for radiotherapy dose calculations: correction of cupping artifacts and conversion of CT numbers to electron density Med. Phys. 35 849-65

Richter A, Hu Q, Steglich D, Baier K, Wilbert J, Guckenberger M and Flentje M 2008 Investigation of the usability of conebeam CT data sets for dose calculation Radiat. Oncol. 342

Siewerdsen J and Jaffray D 2001 Cone-beam computed tomography with a flat-panel imager: magnitude and effects of x-ray scatter Med. Phys. 28 220-31

Varian Medical Systems, Inc. 2008 Customer Release Notes: On-Board Imager Advance Imaging (1.4.04) (Doc-ID $\mathrm{P} / \mathrm{N} \mathrm{KC1404RN)}$

Yang Y, Schreibmann E, Li T, Wang C and Xing L 2007 Evaluation of on-board kV cone beam CT (CBCT)-based dose calculation Phys. Med. Biol. 52 685-705

Yoo S and Yin F 2006 Dosimetric feasibility of cone-beam CT-based treatment planning compared to CT-based treatment planning Int. J. Radiat. Oncol. Biol. Phys. 66 1553-61 